\title{
Metamaterials with negative compressibility - a novel concept with a long history
}

\author{
ATTILA R. IMRE* \\ MTA Centre for Energy Research, POB 49, H-1525, Budapest, Hungary
}

\begin{abstract}
Metamaterials with negative compressibility are a very promising group of novel materials with a wide variety of potential application. A recent model proposed construction of the structures with three-dimensional negative compressibility by utilizing successive destabilization of stable or metastable states and inducing phase transitions mimicking negative compressibility. Here, we would like to show that similar concept is used by the nature and a nice example of this kind of metamaterial can be seen even in a glass of water.
\end{abstract}

Keywords: metamaterials; negative compressibility; spinodal; metastable transitions

(C) Wroclaw University of Technology.

\section{Introduction}

Metamaterials are materials with unusual properties (like negative refractive index), usually not found in nature [1]. In "mechanical metamaterials", the unusual properties are mechanical ones, like compressibility, Poisson-ratio, thermal expansion coefficients, etc. For most of the materials, these quantities are positive, but in some materials (under some conditions), they can be negative. A good example is the Poisson ratio, which is usually positive, but one can construct materials in the lab or even find them in the nature, showing the negative value for this property (auxetic materials) [2-4]. The same is true for the thermal expansion coefficient, for one- or two-dimensional compressibility etc.

Most of the systems showing these properties do not violate (not even virtually) any law of the thermodynamics [5, 6]. Three-dimensional compressibility was always an exception; the positivity of compressibility is per definitionem equal to the stability [7]. Materials which expand under pressure cannot be stable, they would explode, and therefore construction of this kind of system was always a great scientific challenge [8-10], although systems with low-dimensional negative

*E-mail: imre.attila@energia.mta.hu compressibility (like 1D line-compressibility) can be found quite easily [11].

Recently a new type of mechanical metamaterial with three-dimensional negative compressibility has been described. Nicolaou and Motter [12] used the destabilization of metastable or stable equilibrium by applying a finite (not infinitesimal) force in their model. In this way, one might induce a transition, where the jump from the initial to the final state could be described as a negative-compressibility transition. With well-chosen micro- or mesostructure, one can construct a bulk material, where the response function (i.e. the compressibility) for finite, and sufficiently high, pressure change will be always negative, due to the successive transitions. The concept of Nicolaou and Motter can be very useful to design special systems [13]. It should be mentioned here, that similar concept was proposed by Imre et al. [14] using slow transition and the temporal violation of stability criterion.

We would like to show in this short paper, that systems (or materials) showing negative compressibility using this concept are neither novel nor artificial in any way. These kinds of systems do exist since the existence of the Universe, minus that short hectic time, when it was too hot and young for any materials. 


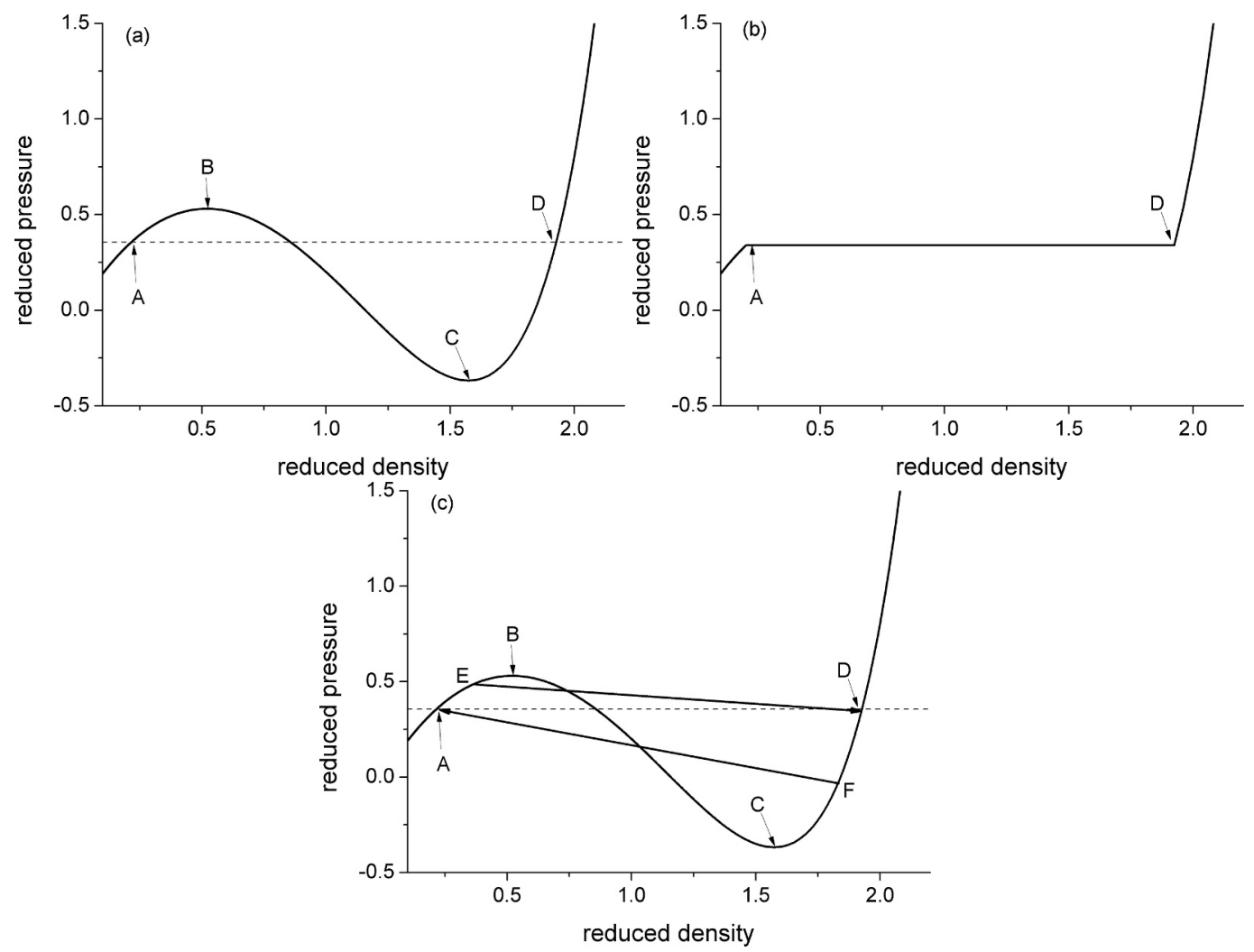

Fig. 1. (a): a complete sub-critical van der Waals loop: stable phases and stability limits are marked, (b): a "loopless" van-der-Waals loop, where metastable and instable "states" are replaced by the Maxwell-line (c) phase transitions from metastable to stable states (from oversaturated vapour to stable liquid, E-D, from stretched liquid to stable vapour, $\mathrm{F}-\mathrm{A}$ ). Transitions along $\mathrm{E}-\mathrm{D}$ and $\mathrm{F}-\mathrm{A}$ lines are mimicking negative compressibility.

\section{Example}

In Fig. 1a, one can see one of the best-known curves of physics and chemistry, the van der Waals loop. Although it was named after van der Waals, who described it mathematically in 1873 [15], it was used for the first time by the Lord Kelvin's older brother, James Thomson [16] at 1871. One can see, that going along the curve from the stable point A (stable vapour with low density) to the stable point $\mathrm{D}$ (stable liquid with high density) one have to go through two regions of metastability $(\mathrm{A}-\mathrm{B}$; oversaturated vapour; and $\mathrm{C}-\mathrm{D}$, superheated or stretched liquids $[7,17,18]$ and, what is more important in this case, an instable region (B-C), where the compressibility would be negative. This continuous transition from A to D along the isotherm is usually handled as an avoidable artefact; in several cases the loop is replaced by the loop-less version, smoothed by the Maxwellconstruction, replacing the whole $\mathrm{A}-\mathrm{D}$ region by a straight line (see Fig. 1b), indicating a zerocompressibility transition. In Fig. 1c, one can see that phase transitions from metastable states to the stable ones (relaxations) are very similar to the ones proposed by Nicolaou and Motter [12]. Explosive boiling of a stretched liquid (from point $F$ to point A) or sudden condensation of oversaturated steam (from point $\mathrm{E}$ to point $\mathrm{D}$ ), represented by the two arrows, can be characterized as negative compressibility transitions. To generate this kind of transition, one should de-stabilize a stable state (like A or D) with a "force" sufficiently high to overcome the energy barrier for the heterogeneous or homogeneous nucleation or reach the stability limit (spinodal) [7, 17]. Here we should mention 
that there are several indications where this kind of loop - together with the negative compressibility part - can be realized experimentally in confined systems (nanopores) [19].

Concerning the experimental existence of a bulk material with negative compressibility, it should be mentioned that since the very beginning of the existence of the van der Waals-loop, it has been proposed that this loop is a quite good description of the flat, finite-width liquid-vapour interfacial region between the equilibrium liquid and vapour [20,21]. Recent simulations describing the inner structure of this interfacial region support this view [22-25]; density and pressure values corresponding to instable states can be found by crossing the interface vertically. Using similar interfacial data, one can even determine the stability limit (corresponding to $\mathrm{B}$ and $\mathrm{C}$ in Fig. 1) of the fluids [22, 23]. In Fig. 2, one can see the interface profile of carbon-dioxide at $270 \mathrm{~K}$ obtained by molecular dynamic simulation [26]. The solid line represents the tangential pressure component (in an $\mathrm{x}$-y-oriented interface, it is $\mathrm{p}_{x x}$ or $\mathrm{p}_{z z}$ element of the pressure tensor inside the interface), while the dashed line is the scalar pressure (one third of the $\left.\mathrm{p}_{x x}+\mathrm{p}_{y y}+\mathrm{p}_{z z}\right)$ [26]; the similarity to the van der Waals loop is undeniable. Although at lower temperatures, the liquid-vapour interface is very thin (for water, at room temperature and at ambient pressure, it is around 3-molecule wide), but close to the critical point, it can be wide enough to be handled as bulk [27]; one can even see it with bare eyes as a grey-milky band between the liquid and vapour phases. In this way, in a glass of water (well, in very hot water) one can find a narrow, but finite width interfacial layer and part of that layer (corresponding to densities between $\mathrm{B}$ and $\mathrm{C}$ in Fig. 1) can be handled as a metamaterial with negative compressibility.

\section{Conclusion}

One can conclude, that the basis of the conception proposed by Nicolaou and Motter [12] (destabilization of metastable equilibrium) is very similar to the conception of a phase transition in a van-der-Waals fluid. The destabilizing force has to

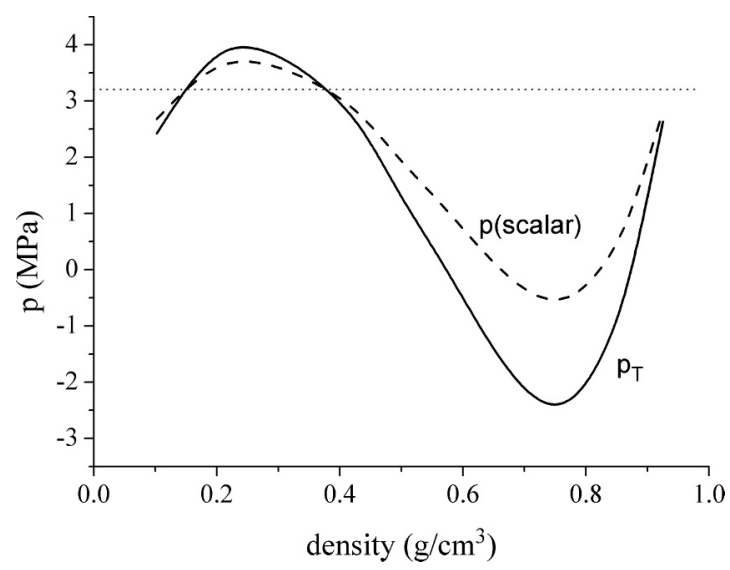

Fig. 2. Smoothed pressure vs. density values within the liquid-vapour interface of carbon dioxide, crossing the interfacial layer from the vapour side to the liquid one [26]. Solid line represents the tangential pressure component (responsible for the surface tension), the dashed one shows the corresponding scalar pressure. Vapour pressure is marked by dotted line.

be big enough to cross the energy barrier for nucleation or to reach the spinodal. In real fluids, the energy barrier for the heterogeneous nucleation (which is the most common process for triggering phase transition) can be few orders of magnitude smaller than the barrier for spinodal transition, therefore nucleation can be more easily utilized than spinodal transition. For water at room temperature, the estimated spinodal pressure is around $-300 \mathrm{MPa}$, while the heterogeneous nucleation limit of "pure" (i.e. distilled) water is just a few MPa below zero (negative pressure means isotropic triaxial stretch) $[7,17,18,28]$. Systems changing from one phase to another (i.e. from liquid to vapour) might - and sometimes do - cross states with negative compressibility and even after the transition, the interface which separates the equilibrium phases keeps these transient conditions, providing a metamaterial-layer (corresponding to the $\mathrm{B}-\mathrm{C}$ region in Fig. 1a), hidden in the liquid-vapour interface. Nevertheless, the theory of Nicolaou and Motter [12], concerning the successive application of the finite "negative compressibility" transition, is a novel and very promising tool to produce smart materials. 


\section{References}

[1] Chen H., Chan C.T., Sheng P., Nat. Mater., 9 (2010), 387.

[2] LAKES R., Science, 288 (2000), 1976.

[3] Wheeler L., GuO C.Y., J. Mech. Mater. Struct., 2 (2007), 1471.

[4] Maruszewsk B.T., Drzewiecki A., Starosta R., ReSTUCCIA L., J. Mech. Mater. Struct., 8 (2013), 403.

[5] Lakes R., Wojciechows Ki K.W., Phys. Status Solidi $B, 245$ (2008), 545.

[6] Gatt R., Grima J.N., Phys. Status Solidi-R, 2 (2008), 236.

[7] Debenedetti P.G., Metastable Liquids: Concepts and Principles, Princeton University Press, Princeton, 1996.

[8] Baughman R.H.,Staftstrom S., Cui C., DantaS S., Science, 279 (1998), 1522.

[9] Barnes D.L., Miller W., Evans K.E., Marmier A., Mech. Mater., 46 (2012), 123.

[10] Gatt R., Carauna-Gauci R., Grima J.N., Nat. Mater., 12 (2013), 182.

[11] Bishop M., Uhoya W., Tsoi G., Vohra Y.K., FeFat A.S., Sales B.C., J. Phys.-Condens. Mat., 22 (2010), 425701.

[12] Nicolaou Z.G., Motter A.L., Nat. Mater., 11 (2012), 608.

[13] Grima J.N., Carauna-Gauci R., Nat. Mater., 11 (2012), 566.

[14] Imre A.R., Drozd-RzoskA A., KraskA T., Rzoska S.J., WoJciechowski K.W., J. Phys.Condens. Mat., 20 (2008), 244104.

[15] VAN DER WAALS J.D., Over de Continuiteit van den Gas - en Vloeistoftoestand (On the continuity of the gas and liquid state), $\mathrm{PhD}$ thesis, Leiden, The Netherlands, 1873.
[16] Thomson J., P. Roy. Soc., 20 (1871 - 1872), 1.

[17] ImRE A.R., MARIS H.J., Williams P.R., (Eds.), Liquids under Negative Pressure, NATO Science Series, Kluwer, Dordrecht, 2002.

[18] El MekKi-Azouzil M., Ramboz C., Lenain J.F., CAUPIN F., Nat. Phys., 9 (2013), 38.

[19] Aguilar-Pineda G.E., JimeneZ-Angeles F., Yu J., Lozada-Casou M., J. Phys. Chem. B, 111 (2007), 2033.

[20] VAn Der WaAls J.D., Verhand. Kon. Akad. Wetensch. Amst. Sec. 1, 1893 (in Dutch; English translation in: J. Stat. Phys., 21 (1979), 197).

[21] BAKKER G., Handbuch der Experimentalphysik; Wien, in: F. Harms, H. Lenz (Eds.), Akad. Verlagsges, Leipzig, 1928.

[22] Imre A.R., Mayer G., Házi G., Rozas R., Kraska T., J. Chem. Phys., 128 (2008), 114708.

[23] Imre A.R., Kraska T., Fluid Phase Equilibr., 284 (2009), 31.

[24] Moore S.G., Wheeler D.R., J. Chem. Phys., 136 (2012), 164503

[25] Werth S., Lishchuk S.V., Horsch M., Hasse H., Physica A, 392 (2013), 2359.

[26] Kraska T., Römer F., Imre A.R., J. Phys. Chem. B, 113 (2009), 4688.

[27] Mayer G., Házi G., PÁles J., Imre A.R., Fischer B., Kraska T., Int. J. Mod. Phys. C, 15 (2004), 1049.

[28] ImRe A.R., Baranyai A., Deiters U., Kiss P.T., KRASKA T., Quinones-Cisneros S.E., Int. J. Thermophys., 34 (2013), 2053. 